\title{
Extraction and Characterization of Natural Cellu- lose Fibers from Sanseveria Trifasciata Plant
}

\author{
Asaye Dessie Wolela* \\ Department of Textile Engineering, Ethiopia
}

ISSN: 2578-0271

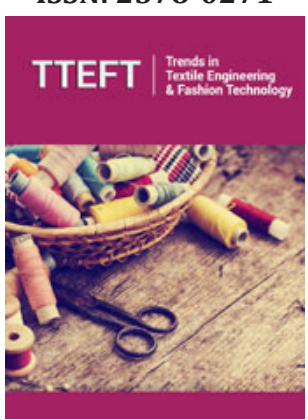

*Corresponding author: Asaye Dessie Wolela, Department of Textile Engineering, Ethiopia

Submission: 海 June 7, 2019

Published: 制June 21, 2019

Volume 5 - Issue 2

How to cite this article: Asaye Dessie Wolela. Extraction and Characterization of Natural Cellulose Fibers from Sanseveria Trifasciata Plant. Advancements Bioequiv Availab.5(2). TTEFT.000609.2019. DOI: 10.31031/TTEFT.2019.05.000609.

Copyright@ Asaye Dessie Wolela, This article is distributed under the terms of the Creative Commons Attribution 4.0 International License, which permits unrestricted use and redistribution provided that the original author and source are credited.

\begin{abstract}
The present study was undertaken to investigate fibers extracted from Sanseveria trifasciata leaves and to analyze their physical behavior of the fiber. The fibre was extracted from the leaves by retting method. Physical and mechanical characterizations were performed on Sanseveria trifasciata fiber (STF): fibre strength, elongation, fiber length, fineness, diameter and moisture content. The functional elements of the fiber were studied using FTIR spectroscopy for better understanding of their behavior. Thus, the above characteristics confirm that this fiber has wide scope in the field of textiles application.
\end{abstract}

Keywords: Natural fibre; Cellulosic fibers; Sanseveria trifasciata plant; Fiber extraction; Characterization

Introduction

Nowadays, the development of environmentally friendly products is at its core point, in order to the constant study for practical solutions to environmental problems and the urgent need to change from non-renewable products. Multiple investigations have been developed in this regard trying to find new materials in order to replace the conventional ones such as plant fibres which have so many advantages like renewable natural resource, biodegradable, cheap skate in cost, good specific mechanical properties and low density [1-4]. Cellulose is a natural bio polymer and can be obtained from a large variety of plant life. The resulting fiber is renewable and biodegradable [5]. Eco friendly, biodegradable and recyclable products are gaining importance in the market which has brought natural fibre in to focus $[5,6]$. The earth is abundantly populated with several varieties of plants that might possibly have a potential to yield valuable fibers, but they are yet to be explored [7]. Long leafy plants seemingly have higher degree of fiber yielding capacity ${ }^{[5]}$. Generally, natural fibers are easily available, easy to extract, easy to handle, light, easy to fabricate, and easily biodegradable $[8,9]$.

There are many fibre yielding plants in Ethiopia, which has wide potential for use in diversified fields, but they remain unexplored so far. The less explored natural fibres belong to leaf fibres. Hence in this work an attempt has been made to extract fibres from Sanseveria trifasciata leaves and it was examined for its structural aspects for textile applications. The Sanseveria trifasciata leaf is found abundantly in our country. Sanseveria trifasciata (Ruscaceae Agavaceae (family) Sanseveria (Genus), Sanseveria trifasciata (Species) is an evergreen herbaceous perennial plant forming dense stands, spreading by way of its creeping rhizome, which is sometimes above ground, sometimes underground. Its stiff leaves grow vertically from a basal rosette. Mature leaves are dark green with light gray-green cross-banding and usually range between $70-90 \mathrm{~cm}$ in length and $5-6 \mathrm{~cm}$ in width [10]. It is commonly called the snake plant (not to be confused with the very similarly named "Snake plant", Nassauvia Serpens), because of the shape of its leaves, or mother-in-law's tongue because of their sharpness $[10,11]$. The plant, which is popularly called snake plant or bowstring hemp, belongs to the family of Agavaceae and is known to grow in Africa, Asia and Florida $[12,13]$. The plant is easy to multiply and cultivate in any surrounding areas even the plant is found in this compound used as decorative purpose. They grow anywhere in anything, in full sun or light shade but thrives well in a moist, fertile soil with a high organic matter content with minimum care [14]. Sanseveria is known variously as bow-string hemp, 
snake plant, zebra lily, mother in law's tongue, cow tongue, leopard lily, devil's tongue, good luck plant, etc. Sanseveria trifasciata is the most common species found cultivated in gardens or pots, particularly Sanseveria trifasciata varlaurentii. The mottled, erect and stiff leaves of Sanseveria are used a great deal in artistic flower arrangements. Sanseveria are a source of white strong elastic fiber commonly used in the manufacture of rope, fishing lines, cordage, fine matting, bowstring, and clothing which can substitute the applications of variety of fiber [9]. Eco-friendliness in textiles is one of the important issues in recent years because textiles are in continuous contact with skin. Nature is blessed with invaluable fiber yielding plants which has wide potential for use in diversified fields, but they still remain in the background. So, this study mainly focuses on the exploration of Sanseveria trifasciata fibre for textile applications. In this investigation an attempt has been made to explore Sanseveria trifasciata fiber for textile applications.

\section{Materials and Methods}

\section{Materials}

The Sanseveria trifasciata leaves were collected from the rural area of Ethiopia. For the purpose of extraction and to characterize the extracted fibers different types of equipment and materials have been used in this investigation.

Fiber extraction: There are different techniques of leaf fibre extraction methods, from these techniques retting and hand scrapping are the major ones.

Hand scraping: In this fibre extraction method a knife or ceramic plate are used, by putting the Sanseveria trifasciata leaf on a plate and scratch repeatedly by using the knife or ceramic plate until the fiber is seen, after that scratch gently or slowly in order to prevent the fibers from damage. Finally, the extracted fiber will be washed by water and dried by sunlight. Hand scraping of the leaves must be done in the first three days after harvesting. If left any longer, the leaves will become dry and the fibers will be difficult to extract. The gathered leaves must be sorted to remove any damaged, diseases or broken leaves. Hand scraping is the easiest and the shortest way of fibre extraction method mechanically, but it is the most tiresome, time consuming and requires much manpower energy (Figure 1 \& 2).

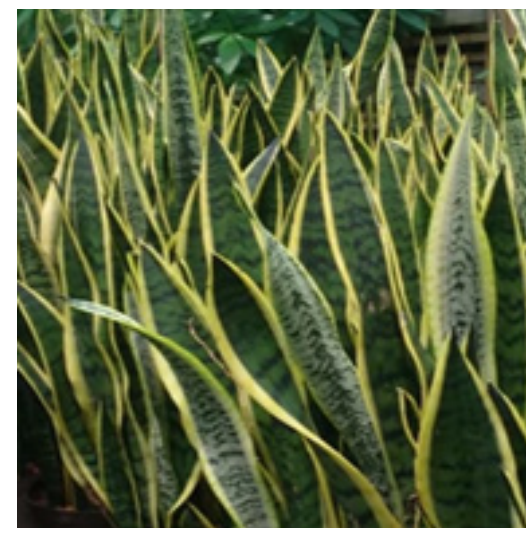

Figure 1: Sanseveria Trifasciata Plant.

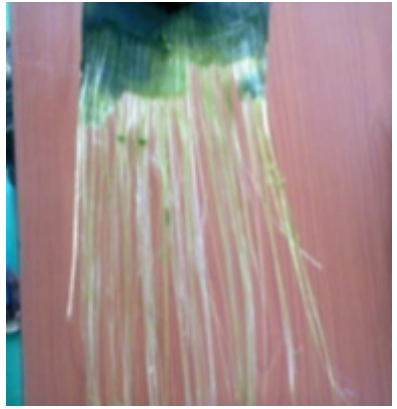

Figure 2: Hand Scraping.

\section{Retting}

In the stagnant water retting the leaves were pounded and made into bundles. These bundles were immersed in stagnant water by placing stones over it to keep the entire leaves submerged for about three weeks. The leaves were then removed, washed and finally dried in sunlight for about 5-7 hours (Figure 3).

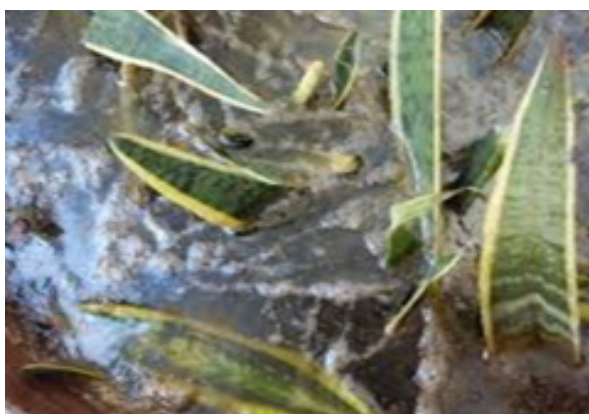

Figure 3: Water Retting.

In the chemical retting methods, the leaf of Sanseveria trifasciata fibre were treated with an alkaline (Sodium hydroxide) solution and allowed to soak in the solution by varying concentration, time, and temperature. Then the fibres are washed with water to remove the excess quantity of $\mathrm{NaOH}$ sticking to the fibres. Finally, the fibers were washed with distilled water and dried in sun. After several trials, the most optimum conditions of fiber extraction were developed based on the yield, length, and strength of fibers obtained. With the optimized conditions the leaf was dipped in 5\% $\mathrm{NaOH}$ solution with a solution to leaf ratio of $25: 1$ at $70{ }^{\circ} \mathrm{c}$ for 60 minutes.

\section{Experimental Procedure (Figure 4)}

\section{Characterization of STF}

The fiber properties of STF were investigated for their potential as a source of textile fibers. The following tests were carried out.

Fibre length: The fibre length was measured using a calibrated metal scale by straightening the fibre over a flat table and the result is expressed in centimeters. Care should be taken that the fibre should not be elongated.

Fiber diameter: Projection Microscope was used to determine the diameter of the fiber. To get accurate results projection microscope micrographs was taken at ten different areas and the average value taken. 


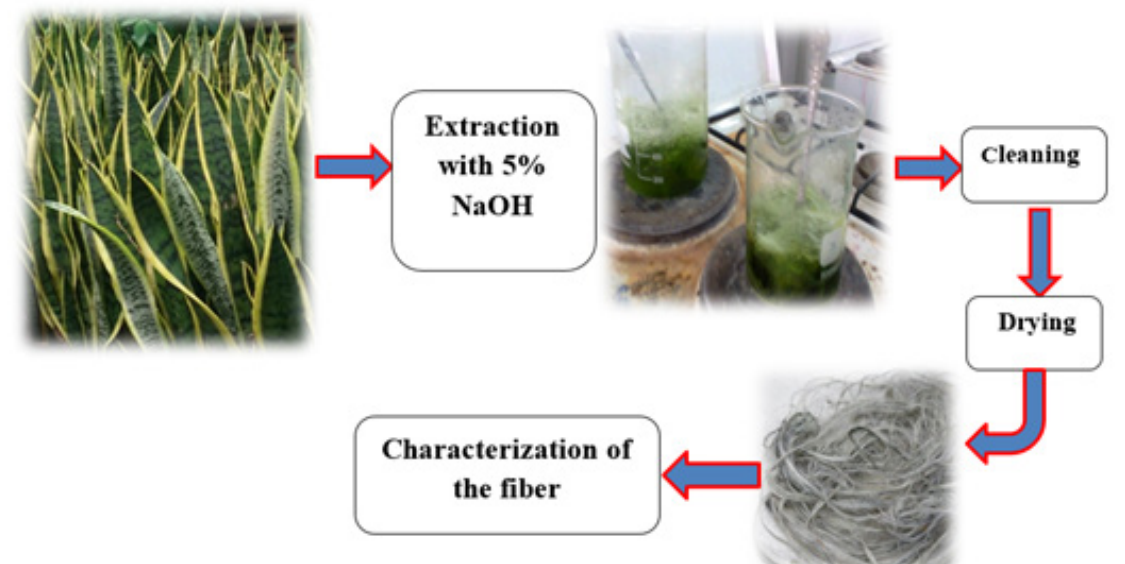

Figure 4: Experimental procedure.

Tenacity and elongation: Tenacity of the fibers was tested using ASTM D-3822:07. The gauge length between the jaws was $15 \mathrm{~cm}$. The preconditioned fiber samples were fixed between two jaws. After the rupture of the fibers the tenacity and elongation were noted.

Fiber fineness: The fiber was tested for its fineness as indicated in ASTM D-1577:07 test method. A fibre bundle was cut into the selected length ( 2 inches) weighed in an electronic balance in $\mathrm{mg}$ to the nearest $0.001 \mathrm{mg}$ and the number of fibres were counted in that bundle. Twenty fibre bundles were randomly selected for testing and the average was calculated.

FTIR study: IR spectra of the samples were recorded using the Perkin Elmer FTIR instrument in the frequency range 4000-500 $\mathrm{cm}^{-1}$ using 20 scans and recorded in the transmittance mode as a function of wave number.

\section{Result and Discussion}

The STF testing reports were studied and the results are reported below.

\section{Fiber length}

The average numbers of fibers extracted from a single leaf of Sanseveria Trifasciata are 772. The lengths of STF were measured manually the average mean value was $90 \mathrm{~cm}$ and the standard deviation and co-efficient of variation are 6.3 and $7 \%$ respectively. The fiber length is considered as one of the essential quality parameter of raw textile material because length to width ratio is the primary requirement of any textile fiber. The range of the fiber length is wide, so it is suitable to produce any count.

\section{Fibre diameter}

Diameter is one of the most important properties of the fiber. The fibre diameter was observed with a projection microscope. The dimension was found to be in the range between $91.85 \mu \mathrm{m}$ $105.96 \mu \mathrm{m}$ with the average value of $97.57 \mu \mathrm{m}$. The standard deviation of the Sanseveria Trifasciata fibers diameter was found to be 4.74 with the coefficient of variation (CV\%) $4.86 \%$. The fibers lack of crimp when seen neither through naked eye nor microscope. The diameter of Sanseveria trifasciata leaf fiber indicates that it is finer than other leaf fibers but coarser as compared with cotton and jute (Figure 5).

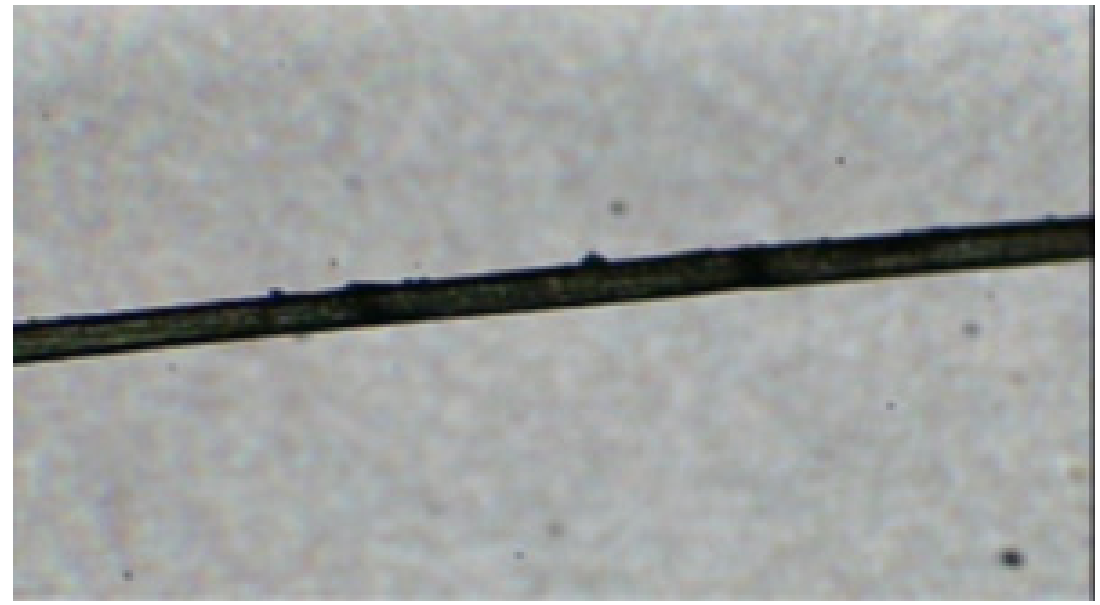

Figure 5: Morphology of Sanseveria trifasciata leaf fibre under 40x magnification microscope. 


\section{Fiber fineness}

Among the three most important fiber characteristics, fiber fineness is one of them. Fineness or micronaire affects both the strength and irregularity of yarn. Fiber fineness also influences the Twist for maximum strength, luster, the drape and handle of fabrics. "The fineness determines how many fibres are present in the cross-section of a yarn of given thickness. The length of a single fiber was measured, and the fiber was weighed. The linear density of the fiber was then calculated in denier units. The mean value of the extracted fiber fineness was found to be 17.65 denier with 4.8 $\%$ as a coefficient of variation.

\section{Tensile properties of STF}

The tensile properties of single STF were determined by a single-fiber tensile testing method as per the specifications of ASTM D-3822:07 standards. Strength and elongation of individual fibers based on the constant rate of elongation were measured. Before carrying out the tensile tests, the sample were conditioned in a standard testing atmosphere of $65 \% \pm 2 \%$ relative humidity and temperature of $21^{\circ} \mathrm{C} \pm 1^{\circ} \mathrm{C}$ for at least $24 \mathrm{hr}$. Tensile strength is the maximum stress caused by a pulling force that a material can withstand without failing. The extracted sansiveria trifasciata leaf fiber was found to have fiber strength of $15.54 \mathrm{~g} / \mathrm{denier}$ and an elongation of $2.6 \%$.

\section{Moisture content and moisture regain}

Most fibers tend to absorb moisture (water vapor) when in contact with the atmosphere. The amount of water absorbed by the textile fiber will depend on the chemical and physical structure and properties of the fiber, as well as the temperature and humidity of the surroundings. The percentage absorption of water vapor by a fiber is often expressed as its moisture regain. The regain is determined by weighing a dry fiber, then placing it in a room set to standard temperature and humidity $\left(21^{\circ} \mathrm{C} \pm 1^{\circ} \mathrm{C}\right.$ and $65 \% \pm 2 \%$ relative humidity $[\mathrm{RH}]$. The moisture regain of the fibers was determined according to ASTM standard method 2654 and the fibre was found to be having $13 \%$ moisture content and a regain of $13.9 \%$.

\section{FTIR characterization}

FTIR analysis is performed to study the organic and inorganic components present in the materials. The IR spectra of STF were shown in below Figure 6.

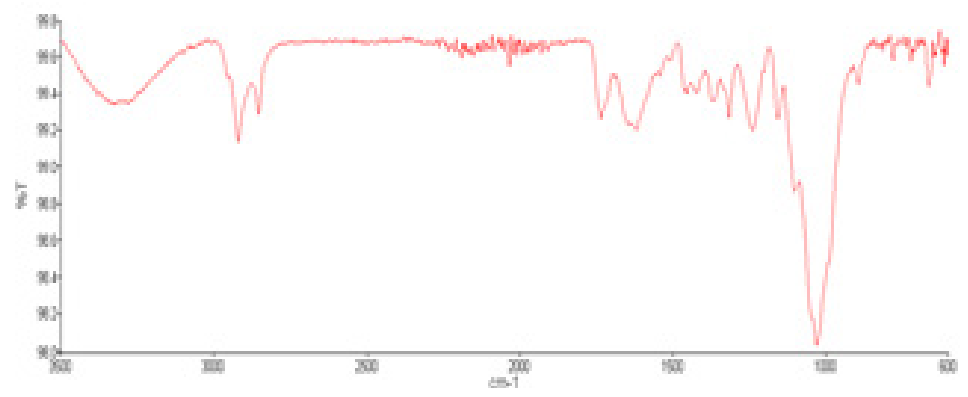

Figure 6: FT-IR analysis.

The above Figure 6 which illustrates the FTIR spectrum of the STF, totally 7 well defined peaks were observed, broad peak at $3432 \mathrm{~cm}^{-1}$ and $2945,1657,1539,1342,1048$ and $608 \mathrm{~cm}^{-1}$.The broad peak observed at $3432 \mathrm{~cm}^{-1}$ corresponds to the stretching vibration mode of intra and intermolecular hydroxyl (-OH) bond of cellulose and the peak $2945 \mathrm{~cm}^{-1}$ corresponding to the asymmetric and the symmetric stretching of methylene (-CH2-) groups in long alkyl chains. These peaks prove the presence of waxes in STF. The absorptions at $1657 \mathrm{~cm}^{-1}$ were due to $\mathrm{O}-\mathrm{H}-\mathrm{O}$ vibrations of water molecules. The bands located in the region $1539 \mathrm{~cm}^{-1}$ are for $\mathrm{CH}_{2}$ of aromatic ring present in the lignin content of the fibre. The peak $1342 \mathrm{~cm}^{-1}$ proved the presence of alcohols, carboxylic acids, esters, ethers and aliphatic compounds. The strong and broad bond at $1048 \mathrm{~cm}^{-1}$ is for C-O of which indicates presence of cellulose in the fiber. The strong bond at $605 \mathrm{~cm}^{-1}$ was assigned to $\mathrm{C}-\mathrm{X}$ of which confirms the presence of bromo-alkanes in the fibers.

\section{Conclusion}

In this paper, natural cellulose Sanseveria trifasciata leaf fibers were investigated for their suitability for textile applications. The Sanseveria trifasciata leaf fibers were extracted from its leaf by retting techniques and the properties of the fibers were characterized. The result showed that the fiber was comparable to other natural fiber such as cotton and flax for most parameters required for clothing such as fineness, moisture, high tenacity and elongation at break. In addition to this, since the Sanseveria trifascita leaf plants were relatively easy to multiply and cultivate in any surrounding areas, they could be considered as an essential alternative source for natural cellulose fibres, and the fiber can be used to make products like apparel and packaging materials.

\section{References}

1. Vandana Balan (2008) Development of preparatory processes for making of banana fibre blended fabrics and their evaluation. SNDT Women's University, Mumbai, India.

2. Samrat Mukhopadhyay, Raul Fangueiro, Yusu Arpac, Ulku Sendurk (2008) Banana fibers variability and fracture behavior. Journal of Engineered Fibres and Fabrics 3(2): 39-45.

3. Fortunati E, Puglia D, Monti M, Santulli C, Maniruzzaman M, et al. (2012) Cellulose nanocrystals extracted from okra fibers in PVA nano composites. Journal of Applied Polymer Science, USA.

4. (2019) J General Engineering 16(3).

5. Hariharashayee D, Akram Abu HM, Aravind S, Mohamed Yasar S, Abdul Azeez M (2018) Investigation of mechanical properties of $\mathrm{KMnO}_{4}$ treated Sansevieria cylindrical fiber reinforced polymer composite. 
International Journal of Advance Research and Development Volume 3(4): 294-298.

6. Vignesh V, Balaji AN, Karthikeyan MKV (2016) Extraction and characterization of new cellulosic fibers from Indian mallow stem: an exploratory investigation. International Journal of Polymer Analysis and Characterization 21(6): 504-512.

7. Kanimozhi M (2011) Investigating the physical characteristics of sansevieria trifasciata fibre. International Journal of Science and Research Publication 1(1): 1-4.

8. Mayandi K, Rajini N, Pitchipoo P, Winowlin Jappes JT, Varada Rajulu A (2016) Extraction and characterization of new natural lignocellulosic fiber cyperu spangorei. International Journal of Polymer Analysis and Characterization 21(2): 175-183.

9. Abral H, Kenedy E (2015) Thermal degradation and tensile strength of sansevieria trifasciata polypropylene composites. IOP Conference Series: Materials Science and Engineering 87: 012011.
10. Ashok Kumar M, Ramachandra Reddy G, Harinatha Reddy G, Chakradhar KVP, Nanjunda Reddy (2011) Mechanical properties of randomly oriented short Sansevieria trifasciata fibre/epoxy composites; International Journal of Fiber and Textile Research 1(1): 6-10.

11. Kanimozhi M (2011) Extraction, fabrication and evaluation of sanseveria trifasciata fiber. Indian Journal of Applied Research 1(2): 97-98.

12. Oguzie EE (2007) Corrosion inhibition of aluminum in acidic and alkaline media by Sansevieria trifasciata extract. Corrosion Science 49(3): 1527-1539.

13. Morton JK (1961) West african lilies and orchids. Longman Green, London.

14. Appell SD (2011) The potted garden new plants and new approaches for container gardens, 1000 Washington Ave, New York, USA, pp. 22-23.

For possible submissions Click below: 